À propos de quelques glissements de sens rocambolesques

\title{
À PROPOS DE QUELQUES GLISSEMENTS DE SENS ROCAMBOLESQUES
}

\author{
Zuzana HILDENBRAND \\ Université Palacký, Olomouc
}

\begin{abstract}
En): The paper deals with the semantic content of three German loan words rocambole, schlass and schnorchel. These three lexical units belong to a set of lexical Germanisms, which have been subjected to a frequency analysis in the French modern written language and then to research as to what extent they are known and used by today's native speakers. These lexical units evince small shifts of meaning compared with their definitions which appear traditionally in the French monolingual dictionaries.
\end{abstract}

Keywords (En): Semantic shift ; German loan words ; Written/spoken language ; Journalistic language ; Etymology ; Sociolinguistics

Mots-clés (Fr): Évolution sémantique ; Emprunts lexicaux à l'allemand ; Langue écrite/parlée ; Langage des journaux ; Étymologie ; Sociolinguistique

\section{Introduction}

Rocambole, schlass et schnorchel, trois mots qui peuvent étonner comme éléments du lexique français, ont un trait en commun : l'origine allemande. En tant que germanismes, ils font partie de l'ensemble des unités qui formaient l'objet de notre recherche doctorale récente, travail qui portait sur ce type de vocabulaire de la langue française, notamment sur la persistance des emprunts à l'allemand dans le lexique français contemporain. ${ }^{1}$ La première phase de la recherche a été consacrée à l'observation de la fréquence absolue de 103 germanismes lexicaux ${ }^{2}$ dans les articles de presse par l'intermédiaire des versions informatisées des

\footnotetext{
${ }^{1}$ Cette recherche a abouti à la rédaction de notre thèse intitulée Emprunts lexicaux à l'allemand dans le français contemporain, Olomouc: FF UP, 2011. (Soutenue en 2012.)

${ }^{2}$ Les 103 unités destinées à l'analyse ont été isolées depuis un ensemble d'environ 400 entrées considérées comme des germanismes dans le $T L F(i)$ suite à un tri minutieux respectant de nombreux critères tels que p. ex. l'étymologie directe/lointaine, l'ancienneté des emprunts (uniquement les germanismes adoptés depuis l'époque de l'ancien haut allemand ont été retenus), la probabilité que tel ou tel lexème puisse faire partie du vocabulaire plus ou moins commun (ont été écarté les archaïsmes historiques, les termes purement scientifiques, trop spécialisés etc.) et d'autres. Les différents registres de langue n'ont pas été pris pour critère décisif pour la constitution du corpus d'étude vu que les expressions soutenues aussi bien que familières peuvent faire partie du vocabulaire courant d'une langue. Évidemment, la méthode de tri pour laquelle nous avons opté, aussi bien que le fait qu'une certaine partie des jugements sur les unités analysées n'a pas pu échapper à notre subjectivité, entraîne parfois quelques hétérogénéités dans l'ensemble des 103 lexèmes retenus : certaines unités tendent plus que d'autres vers le registre de langue populaire, voire argotique (ce qui est entre autres le cas de schlass), certaines se situent à la charnière entre la langue courante et le langage spécialisé, comme schnorchel que nous avons, en début de recherche, supposé plus généralisé qu'il ne s'est révélé être suite aux différentes étapes de notre analyse. Pour plus de détails quant à la classification des emprunts à l'allemand selon nos critères, cf. NAVRÁTILOVÁ (2011: 23-31).
} 
journaux et périodiques Libération, Le Figaro et Le Nouvel Observateur ${ }^{3}$, dans une étendue temporelle comprise entre le $1^{\text {er }}$ janvier 2000 et le 31 octobre $2010 .^{4}$ Lors de la deuxième phase de l'étude, les lexèmes les moins fréquents ${ }^{5}$ ont été soumis à une enquête, effectuée à l'écrit parmi 168 répondants natifs, dans le but de voir dans quelle mesure ce type de lexique est encore connu et utilisé par les locuteurs français de nos jours.

Outre les résultats principalement envisagés, notre recherche a fait ressortir quelques constats inattendus concernant la valeur sémantique des lexèmes en question : dans le cas des trois lexèmes formant l'objet d'étude de notre présente contribution, on observe certaines disparités entre la définition de ceux-ci dans les dictionnaires de la langue française et leur perception et l'usage par les locuteurs actuels. Ce phénomène constaté de manière plutôt marginale lors de notre recherche doctorale, nous nous proposons de l'analyser plus en détail : en partant de l'étymologie de chacun des mots étudiés, nous allons nous consacrer à l'évolution de sa signification dans le temps, telle qu'elle est décrite dans les ouvrages lexicographiques à notre disposition, avant tout dans le $T L F(i)$, et confronter ensuite sa définition officielle avec les résultats de notre enquête, témoignant de l'usage et de la valeur sémantique des unités à l'heure actuelle, afin de pouvoir enfin aborder la question de glissements de sens éventuels.

\section{Rocambole}

La rocambole (s. f.), «plante de la famille des Liliacées, variété d'ail cultivée dans les régions méditerranéennes » et, au sens figuré, « chose sans valeur, futilité. Synon. fam. pacotille », éventuellement « attrait piquant de quelque chose » $(T L F(i))$, est, malgré ses apparences phoniques plutôt exotiques, classée comme un germanisme: selon le $T L F(i)$, le mot est issu du régionalisme allemand Rockenbolle et a été adopté dans le français au cours du XVII ${ }^{\mathrm{e}}$ siècle. (Ibid.)

Suite au dépouillement des archives électroniques des trois journaux mentionnés, nous nous sommes subitement rendue compte que la fréquence du mot rocambole dans le français écrit du début du $\mathrm{XXI}^{\mathrm{e}}$ siècle est particulièrement faible : dans le corpus pratiquement inquantifiable qu'offrent les archives informatisées des trois journaux, comportant des centaines de milliers de textes, l'unité recherchée n'est apparue que dans 26 articles et encore, uniquement 2

\footnotetext{
${ }^{3}$ Pour étudier la persistance des germanismes dans le français écrit, nous avons choisi de nous baser uniquement sur des textes journalistiques parce que nous considérons qu'ils reflètent le mieux l'état actuel d'une langue écrite générale. À la différence de la recherche sur Internet tout court, nous sommes épargnés des simples transcriptions de la langue parlée, c.-à-d. de la langue des forums de discussions, du chat etc., nous nous trouvons face à la langue soutenue, considérée comme correcte à l'époque donnée, mais pas trop artificielle, pas trop fleurie puisqu'elle doit être comprise par le public le plus large possible et doit lui faire appel. De plus, les textes journalistiques assurent une grande diversité thématique mais ne risquent pas d'être trop spécialisés comme les textes scientifiques, ils reflètent aussi des nouveautés langagières mais ne sont pas autant fantaisistes et détachés de la langue courante que peuvent l'être certains textes littéraires.

${ }^{4}$ Pour plus de précisions quant à la formation du corpus de recherche, cf. NAVRÁtILOVÁ (2011 : 45$63)$.

${ }^{5}$ Ceux dont la fréquence en français écrit se situait entre 0 et 30 .
} 
occurrences correspondaient au sens recherché. Ceci était dû à deux facteurs : d'abord, les moteurs de recherche ne respectant pas les majuscules/minuscules, nous avons fréquemment eu affaire à Rocambole en tant que nom propre fictif littéraire ; nom d'un personnage des romans de Ponson du Terrail. ${ }^{6}$ Ensuite, les systèmes de recherche de ce type faisant souvent des corrections automatiques des entrées, il n'était pas rare qu'en cherchant le substantif rocambole, on obtienne des résultats pour l'adjectif dérivé rocambolesque.

Les auteurs du TLF(i) définissent l'adjectif rocambolesque comme « invraisemblable, plein de péripéties extraordinaires » et quant à son étymologie, ils l'expliquent comme suit :

Dér. de Rocambole, n. du héros aux aventures extraordinaires des romans-feuilletons de P.A. Ponson du Terrail, parus à partir de 1859. Le n. de Rocambole a peut-être été tiré par cet écrivain de rocambole* « attrait piquant de quelque chose». Cf. rocambolade "farce littéraire dans le goût des Exploits de Rocambole de Ponson du Terrail » (1867, DelvaU). (TLF(i))

À l'origine de toutes ces ramifications sémantiques du mot serait donc la plante au goût piquant, celle-ci aurait inspiré P. du Terrail pour créer un héros littéraire du même nom, dont le caractère (et le caractère des aventures que ce personnage vit) mènerait enfin vers la dérivation adjectivale rocambolesque.

Avec ce qualificatif, on se trouve face à un des mots extrêmement populaires de nos jours, du moins au niveau du jargon journalistique, connu pour son recours typique aux « expressions préfabriquées » (UVÍROVÁ, 2005 : 255), aux «imagesclichés » qui aboutissent souvent à des « tics journalistiques » et des « modismes » (ibid. : 256). Les quelques occurrences de rocambolesque qui se sont mélangées plutôt par accident parmi nos attestations de rocambole ne représentent qu'un ensemble minuscule comparé aux centaines d'occurrences qui s'affichent si l'on entre l'adjectif lui-même dans les moteurs de recherche. Par ailleurs, non seulement ce mot surgit fréquemment dans les articles de presse mais on peut également l'entendre très souvent à la télévision, p. ex. de la bouche des commentateurs sportifs lors de la retransmission d'un match. Il est fort possible que les journalistes qui se servent du mot ne connaissent même pas sa signification exacte, sans parler de leurs connaissances sur l'origine de celui-ci, mais c'est peutêtre entre autres l'effet phonique du mot qui les amène à en abuser. Sur le site de Libération, il y a cinq ans, il était déjà possible de trouver un article dénonçant l'utilisation démesurée de cette expression :

«Une libération vraiment rocambolesque. » Reconnaissons-le : nous autres journalistes, radios, télés ou autres - quand il s'agit de raconter des faits sur lesquels nous avons peu d'informations (ou un gros doute) - n'hésitons jamais à ressortir un bon vieux cliché. Ainsi de l'inusable « rocambolesque». Une évasion est rocambolesque quand on ne connait pas encore les détails de son déroulement. Idem d'une libération d'otages. En général, plus le temps passe, plus on en apprend. Et moins c'est rocambolesque. Spectaculaire, tout au plus. (POURQUERY, 2008) $)^{7}$

6 Larousse Encyclopédie [online]. [cit. 02/02/2012]. Cf. Éditions Larousse. Larousse.fr. Encyclopédie, Paris, http://www.larousse.fr/encyclopedie.

7 Rocambolesque [online]. [cit. 31/01/2012]. Cf. http://www.liberation.fr/week-end/010184790rocambolesque. 
Pour résumer, on peut donc constater que dans le français écrit de nos jours, l'unité lexicale rocambole est peu fréquente, à la différence du cliché rocambolesque qui non seulement a dû se lexicaliser il y a longtemps, vu qu'il a déjà sa place dans un ouvrage lexicographique aussi réputé que le Trésor de la langue française, mais qui de plus est toujours usité près de vingt ans après la publication de celui-ci. ${ }^{8}$

Les constats que nous venons de formuler par rapport à la situation du mot rocambole dans le français écrit moderne, trouvent-ils des parallèles au niveau de la connaissance et de l'emploi de cette unité lexicale par les locuteurs contemporains?

Notre recherche a été, comme on l'a déjà abordé, suivie d'un questionnaire distribué parmi 168 natifs choisis au hasard, de différentes tranches d'âge, de différents sexes, provenant de diverses régions françaises, exerçant divers métiers et ayant des notions de l'allemand et des relations avec les Allemands plus ou moins (ou pas du tout) développées. ${ }^{9}$ Pour chaque unité étudiée, il a été demandé de cocher une des quatre cases représentant différents degrés de familiarisation avec le mot en question. Les catégories ont été intitulées comme suit :

a) Je ne connais pas

b) Déjà entendu mais pas sûr de la signification

c) Je connais mais je n'utilise pas

d) Je connais et j'utilise

Outre cette classification sous une de ces quatre catégories, nous avons exigé pour chaque mot une brève explication de sa signification. (Sauf si, bien sûr, le répondant cochait la case «Je ne connais pas ».) Le but de cette rubrique n'était pas d'obtenir une définition exacte des unités mais de vérifier si la case cochée correspondait à la réalité. ${ }^{10}$

L'enquête a donné les résultats suivants : $46 \%$ des interrogés classifient le mot rocambole comme inconnu, 33 \% l'ont déjà entendu mais ne sont pas sûrs de sa signification, $15 \%$ affirment qu'ils le connaissent mais ne l'utilisent pas et $6 \%$ s'en servent. Dans ce sens, il est possible d'observer une corrélation avec les résultats de la première partie de notre étude : le substantif rocambole n'est pas à compter parmi les plus connus et les plus fréquents.

En revanche, c'est la rubrique «Je crois que le mot veut dire... » de notre questionnaire qui nous amène aux constats quelque peu surprenants : outre certaines définitions erronées qu'il a fallu écarter (entre autres plusieurs fois la définition «fruit» qui découle probablement de la confusion avec carambole), nous avons eu droit aux interprétations telles que : «sorte d'ail » $(13,3 \%)$, « chose sans valeur» $(4,5 \%)$, «personnage littéraire» $(13,3 \%)$, mais aussi,

\footnotetext{
${ }^{8}$ Le $T L F$ a été publié entre 1971 et 1994.

${ }^{9}$ Tous les aspects énumérés faisaient partie du questionnaire. Le degré de familiarisation des sujets avec le vocabulaire d'origine allemande a été étudié en rapport avec leur âge, leur sexe, leur origine et l'endroit où ils vivent actuellement, leur profession, leurs connaissances en langue allemande, leurs rapports envers la nation allemande etc. Cf. NAVRÁTILOVÁ (2011 : 112-135).

${ }^{10}$ Pour plus de précisions sur la méthodologie de la composition de l'enquête, voir NAvRátiLová (2011: 105-112).
} 
majoritairement, «aventure/ histoire/ péripétie surprenante/ invraisemblable/ ridicule/ farfelue/ bizarre» (éventuellement, dans quelques cas ponctuels, « acrobatie/ pirouette ») (68,9\%), et cela entre autres dans les catégories c) et d) $\mathrm{du}$ questionnaire. Les répondants ont d'ailleurs souvent fait, avant tout dans la catégorie b), un rapprochement avec l'adjectif rocambolesque; soit ils confondaient les deux unités, soit ils considéraient rocambole comme un adjectif, soit ils affirmaient que rocambole était selon eux issue de rocambolesque.

Cette prédominance de quasiment $70 \%$ de définitions du substantif rocambole qui ne figure pas dans les dictionnaires du français et qui relève d'un rapport évident avec l'adjectif dérivé rocambolesque, peut naturellement pour une grande partie être due à une simple confusion accidentelle des mots en question par les interrogés. Outre cela, on peut néanmoins se demander si une nouvelle évolution sémantique de rocambole, par une nouvelle substantivation à partir de l'adjectif dérivé, n'est pas en train de se produire.

\section{Schlass}

Un autre mot considéré comme emprunt à l'allemand, moins spectaculaire que rocambole mais qui, lui aussi, est soupçonné de subir un léger glissement de sens, du moins par rapport à son sémantisme indiqué dans les dictionnaires du français, est l'adjectif schlass. Rangé d'habitude dans le registre de langue familier, populaire voire argotique, il est défini dans le $T L F(i)$, de même que dans d'autres dictionnaires de la langue française tels que Larousse 2010 ou Le Nouveau Petit Robert et dans les dictionnaires de l'argot de COLIN (1994) et ESNAULT (1965) simplement comme « ivre », éventuellement « soûl ». ${ }^{11}$ Les deux derniers et le $T L F(i)$ mentionnent plusieurs variantes du mot, que ce soit la forme féminine schlasse ou plusieurs variantes graphiques schlâss(e), chlass(e), chlâss(e), slaze, slass(e), COLIN (1994) et ESNAULT (1965) citent aussi chlassic, chlassig de sens identique et ESNAULT (1965) fait noter même quelques dérivés : chlasser, slasser (« enivrer »), schlassiquer («s'enivrer»), déslasé (« désoulé »).

Il est curieux que schlass figure dans le $T L F(i)$ comme « empr. à l'all. région. schlass », avec la remarque que «le mot n'est pas att. ds E. MARTIN, H. LIENHART, Wörterbuch der elsässischen Mundarten »; chlass(e), traité à part, y est défini en tant que « empr. à l'all. schla $\beta$ « mou, fatigué » (Der SprachBrockhaus), terme alémanique attesté en suisse-allemand, en souabe et en alsacien ( $F E W$ t. 17, p. 41b) et qui a pénétré en France par l'intermédiaire de l'alsacien ». COLIN (1994), de même que Larousse 2010 et Le Nouveau Petit Robert considèrent toutes les variantes du mot comme emprunts à l'allemand et ESNAULT (1965) marque « ETYM. Allem. et alsacien schlass, schlassig ». Le mot coexisterait-il donc en parallèle en allemand et en alsacien, sans qu'on puisse dire lequel des deux est l'étymon de l'emprunt en français ? Ou bien le transfert lexical se serait-il opéré indépendamment par l'intermédiaire des deux langues sources ?

Quoi qu'il en soit de l'étymologie exacte du mot, toutes les sources lexicographiques qui se prononcent sur la signification de celui-ci dans la langue

${ }^{11}$ Larousse 2010 définit le mot de manière plus élégante : «Fam. Qui est dans un état d'ébriété avancé. » 
d'origine indiquent qu'en allemand (ou en alsacien), le mot porte la signification «mou, fatigué », alors que dans la langue cible, c.-à-d. en français, schlass veut dire « ivre ». Nous allons voir que notre recherche remet cette interprétation univoque du mot en question.

Nous avons soumis le substantif schlass au même procédé de recherche que le lexème rocambolesque. Parmi les rares occurrences de ce mot dans les articles de presse étudiés, dont il a fallu écarter encore presque la moitié qui correspondait au substantif masculin homographe schlass (synonyme familier de «couteau ${ }^{12}$ ), nous n'avons relevé que 5 occurrences du sens recherché. ${ }^{13}$ Il est donc évident que dans le français écrit contemporain, le mot en question est très peu fréquent. Il semble être trop familier pour paraître dans les textes journalistiques et doit être réservé davantage à la langue parlée : $29 \%$ des répondants affirment le connaître sans l'utiliser et $12 \%$ disent s'en servir, ${ }^{14}$ ce qui n'est pas un pourcentage négligeable. $^{15}$

Comme dans le cas de rocambole, c'est néanmoins avant tout l'interprétation de l'unité qui est intéressante : en mettant de côté des fausses définitions $(8,5 \%$ par rapport au substantif schlass), on constate que seulement 25,6\% des définitions obtenues correspondent à «ivre (mort), saoul, bourré ». Dans 65,9\% des cas, le mot est interprété comme «fatigué, épuisé, fourbu, crevé, $\mathrm{KO}$ ». Quelques-uns ont noté les deux définitions.

Nous considérons qu'il est improbable qu'il s'agisse d'une évolution sémantique française se rapprochant de la signification originale allemande, postérieure à l'adoption de l'unité dans la langue emprunteuse. On peut plutôt supposer que l'interprétation du mot proche de l'étymon allemand est due aux aspects diatopiques qui se reflètent dans notre enquête. En analysant les réponses en détail, on se rend compte que le critère géographique joue effectivement un rôle : $63 \%$ des répondants ayant bien défini le mot schlass viennent des régions que nous avons décidé de prendre pour géographiquement marquées, c.-à-d. des régions du Nord-Est de la France, proches de l'Allemagne, de la Suisse ou d'autres

\footnotetext{
${ }^{12}$ Mot d'origine obscure, peut-être emprunté à l'anglais slasher, cf. TLF(i).

${ }^{13}$ Quatre des cinq attestations sont apparues dans le journal Libération. Lors de notre recherche, nous avons souvent observé le phénomène de prédominance des occurrences dans les textes de ce journal, avant tout lorsqu'il s'agissait des unités lexicales propres à la langue parlée. Ce fait semble se justifier par le style «écrit-parlé » qui serait caractéristique pour Libération. (Larousse, Encyclopédie [online]. [cit. 02/02/2012].

Cf. http://www.larousse.fr/encyclopedie/oeuvre/Lib\%C3 \%A9ration/129815.)

${ }^{14}$ Contre quand même $48 \%$ de réponses complètement négatives et $11 \%$ de réponses de type « Déjà entendu mais pas sûr de la signification ».

${ }^{15}$ Le fait qu'il s'agisse d'un mot de registre populaire/argotique, incite à se poser des questions quant au conditionnement de la connaissance et de l'usage de celui-ci par l'âge ou le sexe des interrogés. Or, ces aspects ne se sont pas montrés décisifs : parmi les 103 unités analysées, schlass fait partie des mots plus connus et plus utilisés tantôt par les répondants les plus familiarisés avec les germanismes (majoritairement hommes d'âge moyen ou élevé, possédant un degré d'études supérieur et ayant quelques notions en allemand), tantôt par les locuteurs les moins familiarisés avec les germanismes (majoritairement, si l'on simplifie au maximum, jeunes femmes). Cf. NAVRÁTILOVÁ (2011 : 130132).
} 
pays germanophones. ${ }^{16}$ Même au niveau des différentes interprétations de schlass par différents répondants, on constate une prédominance de la signification «fatigué » chez ceux qui vivent dans ces régions frontalières. Les pourcentages résultants ne sont cependant pas parfaitement univoques :

\begin{tabular}{|l|c|c|c|}
\hline \multicolumn{2}{|c|}{ «fatigué » } & \multicolumn{2}{c|}{ «ivre » } \\
\hline Nord-Est & autres régions & Nord-Est & autres régions \\
\hline $48 \%$ & $27 \%$ & $15 \%$ & $10 \%$ \\
\hline
\end{tabular}

Certes, le sémantisme « fatigué » prévaut chez les locuteurs d'origine marquée. Cependant, notons que globalement, même chez ceux qui viennent de tous les autres coins de l'Hexagone, cette signification du mot est majoritaire par rapport à la signification «ivre », prônée comme exclusive de manière unanime par les dictionnaires de la langue française. Naturellement, entre l'état d'ivresse et l'état de fatigue, il y a un lien étroit et celui qui est ivre ressemble forcément à quelqu'un de fatigué. Toutefois, cette disparité entre l'existence du mot en tant que lexème d'un dictionnaire et la vraie vie de celui-ci en tant qu'unité du lexique au service des locuteurs français contemporains nous semble digne d'être remarquée.

\section{Schnorchel}

Pas plus courant que rocambole et schlass et quasiment méconnu est le germanisme schnorchel (s.m.), «tube de prise d'air en surface permettant aux sous-marins, en faible plongée, d'utiliser leurs moteurs diesel ou à des chars immergés d'aspirer l'air frais et de rejeter les gaz d'échappement » $(T L F(i))$.

Dans les articles de presse dépouillés, le mot n'est apparu que 3 fois et quant à sa position dans le lexique actuel des locuteurs natifs, uniquement $2 \%$ des répondants l'utilisent et $4 \%$ le connaissent sans s'en servir. $4 \%$ affirment l'avoir déjà entendu mais ignorent sa signification, alors que pour $90 \%$ des enquêtés, le mot reste complètement étranger. Bien qu'il ne s'agisse pas d'un terme scientifique et que nous ayons présupposé une certaine généralisation de la connaissance de cet outil de plongée dans la population française moderne, la notion reste visiblement confinée dans le langage spécialisé des sous-marins, ou bien elle est peut-être évincée par l'expression tuba, courante de nos jours.

Parmi les sujets peu nombreux qui ont, malgré cette méconnaissance générale, proposé une définition du mot, nous avons toutefois remarqué une petite particularité : à part la définition correcte, correspondant plus au moins à celle du $T L F(i)$, nous avons plusieurs fois eu droit à l'explication «pot d'échappement» ou « cheminée », et cela même en combinaison avec la case «Je connais et j'utilise » cochée. Évidemment, dans ce cas l'échantillon des interprétations du mot est beaucoup trop restreint pour que l'on puisse en tirer des conclusions incontestables et il peut tout simplement s'agir de confusions ponctuelles qui coïncident par hasard. Pourtant, dans le contexte des évolutions sémantiques que nous venons

\footnotetext{
${ }^{16}$ Il faut néanmoins prendre en considération que l'échantillon de personnes ayant répondu à l'enquête a été très inégal au niveau diatopique : quasiment $45 \%$ des interrogés sont d'origine lorraine, plus de $50 \%$ vivent en Lorraine à l'heure actuelle. Pour des données plus précises, cf. NAVRÁTILOVÁ (2011: 115).
} 
d'aborder, il n'est pas sans intérêt de mentionner entre autres ce petit glissement de sens de schnorchel, surtout que dans le cas de celui-ci, il n'est pas possible d'en chercher la cause dans l'adoption de la signification originale allemande. N'auraiton pas affaire à un léger élargissement de sens du mot?

\section{Le sort rocambolesque des emprunts à l'allemand dans le français contemporain}

Comme nous avons pu le voir, les germanismes rocambole, schlass et schnorchel, bien que très différents l'un de l'autre, se caractérisent tous les trois par une disparité plus ou moins flagrante entre leur définition officielle donnée par les ouvrages lexicographiques français et la signification que leur donnent les usagers de la langue française contemporaine. Dans le cas de rocambole, nous constatons l'apparition d'un nouveau sémantisme «aventure/ histoire/ péripétie surprenante/ invraisemblable/ ridicule/ farfelue/ bizarre », né certainement de l'usage abusif de l'adjectif cliché rocambolesque. Pour schnorchel, un glissement de sens se laisse affirmer avec beaucoup moins de certitude, néanmoins, plusieurs définitions correspondant non pas à un outil de sous-marin mais à une cheminée ou un pot d'échappement se font remarquer. Enfin schlass connaît, dans le français contemporain, une utilisation plus proche de celle de sa langue d'origine que de celle de la langue emprunteuse : par les locuteurs français, le mot est défini majoritairement comme «fatigué » plutôt que «ivre », seule définition proposée systématiquement par les dictionnaires français. Dans ce cas, nous serions néanmoins tentée d'attribuer cette disparité plus aux influences diatopiques qu'à un glissement sémantique récent.

Si l'on voulait espérer qu'avec le temps, nos hypothèses sur les sémantismes nouvellement acquis des trois mots étudiés seront confirmées par une lexicalisation parfaite qui aboutira à une réélaboration des entrées concernées dans de nouveaux dictionnaires, ou au contraire qu'elles se montreront éronnées, notre espoir d'être témoin de cette preuve serait certainement vain. Par l'intermédiaire des deux étapes de notre recherche doctorale, étudiant les 103 germanismes, il est à constater que globalement, les emprunts à l'allemand appartiennent de nos jours aux parties du lexique français qui sortent de l'usage, voire se trouvent déjà en voie de disparition. Pour les trois unités lexicales en question, avec leur fréquence minuscule et leur degré de connaissance plus ou moins négligeable, ceci n'est pas moins valable. A l'avenir, en consultant de nouveaux dictionnaires du français, on peut donc plus probablement s'attendre à ce que ces lexèmes n'y figurent plus du tout, plutôt qu'à une élaboration approfondie de leurs notices. Le seul mot qui pourrait peut-être encore nous surprendre est rocambole; à condition que la popularité du rocambolesque ne cesse de durer. 


\section{BIBLIOGRAPHIE}

COLIN Jean-Paul ; MÉvEL Jean-Pierre ; LECLÈRE, Christian (1994), Dictionnaire de l'argot, $2^{\mathrm{e}}$ éd., Paris, Larousse. [1 ${ }^{\mathrm{èr}}$ édition, 1990]

Éditions Larousse, Larousse.fr. Encyclopédie, Paris, [http://www.larousse.fr/encyclopedie, cit. 02-02-2012].

ESNAULT Gaston (1965), Dictionnaire historique des argots français, Paris, Larousse.

IMBS Paul; QUEMADA, Bernard (dir.) (1971-1994), Trésor de la Langue Française. Dictionnaire de la langue du XIX et du XX siècle (1789-1960), 16 volumes, Paris, Éditions du CNRS/Gallimard. Trésor de la Langue Française informatisé [http://atilf.atilf.fr/tlf.htm, cit. 04-02-2012].

JEUGE-MAYNART Isabelle (dir.) (2009), Le petit Larousse illustré 2010, Paris, Larousse.

NAVRÁTILOVÁ Zuzana (2011), Emprunts lexicaux à l'allemand dans le français contemporain, thèse non éditée, Olomouc, FF UP.

Nouvel Observateur du Monde, SA/ NTT Europe Ltd. Le Nouvel Observateur, Paris, [http://tempsreel.nouvelobs.com/, cit. 04-02-2012].

POURQUERY Didier (2008), « Rocambolesque », in : Libération (Week-end, le 05/07/2008) [http://www.liberation.fr/week-end/010184790-rocambolesque, cit. 31-01-2012].

REY Alain ; REY-Debove, Josette (dir.) (1993), Le Nouveau Petit Robert, édition entièrement revue et amplifiée du Petit Robert, Paris, Dictionnaires Le Robert.

SARL Libération. Libération.fr, Paris, [http://www.liberation.fr/, cit. 04-02-2012].

Société du Figaro, SAS/ SDV Plurimédia. Le Figaro.fr, Paris/Strasbourg, [http://www.lefigaro.fr/, cit. 04-02-2012].

UVÍROVÁ Jitka (2005), «Le Beaujolais nouveau est arrivé ! », in : Romanica Olomucensia XV (Acta Universitatis Palackianae Olomucensis, Facultas Philosophica, Philologica 87), Olomouc, FF UP, p. 255-261. 